\section{Managementsysteme in der Wirtschaft}

von Michael F. Jischa, Technische Universität Clausthal

In Wirtschaftsunternehmen ist Technik schon immer bewertet worden, bislang idoch überwiegend nach den Kriterien Funktionalität und Sicherheit sowie Wirtschaftlichkeit. Angesichts des allseits akzeptierten Leitbildes Nachhaltigkeit ist die Zielfunktion für Unternehmen komplexer geworden. Sowohl aus politischer wie auch aus unternehmerischer Sicht muss die zukünftige Entwicklung so gestaltet werden, dass ökonomische, ökologische und gesellschaftliche Zielsetzungen gleichrangig angestrebt werden. Bereits heute werden Umweltkosten in die Kosten-Managementsysteme der Unternehmen integriert. Ausgehend von dem etablierten QualitätsManagementsystem ist das UmweltManagementsystem entstanden. Dies beinhaltet schon weitgehend standardisierte Instrumente wie Life-Cycle-Assessment (LCA), deutsch Ökobilanzen genannt, sowie das Ressourcen-Management. Weitere Managementsysteme kommen hinzu, so etwa das Risiko-Management. Der vermutlich nächste Schritt wird hin zu einem umfassenden Nachhaltigkeits-Managementsystem gehen. Hier treten alle in der TADiskussion bekannten Problemstellungen wie etwa Abgrenzung und Bewertung, Aggregation und Indikatoren, Quantifizierung von weichen (nicht-technischen, nichtökonomischen) Bewertungsgrößen, unterschiedliche Zeitskalen, Integration von unscharfem oder auch Nicht-Wissen sowie Umgang mit Werten und Wertkonflikten auf. Fazit: TA ist Nachhaltigkeits-Management aus Sicht der Wirtschaft.

\section{Zum Schwerpunktthema}

Die Wahl des Schwerpunktthemas begrüße ich außerordentlich. Ich hätte es mir schon früher gewünscht, weil ich die einseitige Fokussierung von TA auf Politikberatung immer für einen Fehler gehalten habe. Das hat der TABewegung nicht gut getan und der Etablierung von TA als anerkannte wissenschaftliche Disziplin eher geschadet als genützt. Dies lässt sich sehr deutlich der vehementen Erwiderung von H. Fürstenwerth in Heft 4/2000 dieser Zeitschrift entnehmen, die eine Antwort auf den Diskussionsbeitrag von A. Grunwald in Heft 3/2000 gewesen ist.

Es ist zu vermuten, dass die aus meiner Sicht sehr lesenswerten Anmerkungen von H. Fürstenwerth die Herausgeber zu diesem Schwerpunktthema angeregt haben. Wie schön, dass H. Grunwald mit seinem Beitrag in Heft 3/2000 den Stein ins Wasser geworfen hat. Weitere Wogen der Erregung, zustimmende wie kontroverse, werden folgen. Die Beiträge hierzu von A. Zweck, von H.-J. Luhmann sowie von A. Grunwald im Diskussionsforum des Heftes 1/2001 geben einen Vorgeschmack darauf. Ich bin sehr gespannt auf die Beiträge hierzu in diesem Heft!

Die frühe Verengung von TA auf Politikberatung ist aus der kurzen Geschichte der TA nachvollziehbar. Aber mussten wirklich nahezu drei Jahrzehnte verstreichen, bis in etablierten TA-Zirkeln die Einsicht greifen sollte, TA könnte sehr viel mehr leisten als „nur“ Politikberatung? Wie wetterwendisch agiert doch die Politik im Gegensatz zu der Wirtschaft, deren Rationalität viel kalkulierbarer und somit verlässlicher ist.

Ich hege den Verdacht, dass die frühen TA-Zirkel zu stark von der ersten Kultur der Geistes- und Gesellschaftswissenschaften dominiert waren, deren Definitionsmacht zusammentraf mit ihrer Aversion (oder auch nur Unkenntnis) gegenüber der Wirtschaft. Anders sind die langen akademischen Diskussionen etwa zu der Frage, wie denn nun TA übersetzt werden sollte, kaum nachzuvollziehen.

Ich plädiere dafür, dass die Vertreter der Zweiten Kultur, der Natur- und Ingenieurwissenschaften, sowie insbesondere die Manager der Wirtschaft, welcher Disziplin sie auch immer angehören mögen, deutlicher als bisher in die Diskussion über TA eingreifen. In diesem Sinne möchte ich meinen Beitrag verstanden wissen.

\section{Managementsysteme in der Wirt- schaft}

In der Wirtschaft wird ständig auf der Basis von Bewertungen entschieden. So ist auch Technik schon immer bewertet worden, also 
Technikbewertung betrieben worden, ohne jedoch diesen Begriff zu verwenden. Die Bewertungskriterien waren klar und eindeutig. Sie waren technischer Art, wenn es um Fragen der Funktionalität und Sicherheit ging. Und sie waren betriebswirtschaftlicher Art, denn technische Produkte müssen sich am Markt behaupten können.

Bewertungen dieser Art werden in der Wirtschaft nach wie vor vorzugsweise von Ingenieuren und von Betriebswirten vorgenommen. Aus dem Kreis der Naturwissenschaftler sind hauptsächlich Chemiker und weniger Physiker anzutreffen, denn im Gegensatz zur Chemischen Industrie gibt es keine eigenständige Physikalische Industrie. Physiker sind jedoch aufgrund ihrer Ausbildung hervorragend geeignet, entstehende Lücken (etwa in Elektronik, in Materialforschung, in Informatik usw.) zu füllen.

Welches sind nun generelle Bewertungsprobleme? Es sind dies Abgrenzung und Bewertung in Form von aussagefähigen, meist hochaggregierten Bewertungsgrößen, den Indikatoren. Die Suche nach einfachen, signifikanten und aussagekräftigen Bewertungsgrößen bewegt sich stets zwischen zwei Extremen: Das Einfache ist theoretisch falsch, und das Komplizierte ist praktisch unbrauchbar. „We need quick and dirty methods", so hat sehr treffend ein englischer Manager in einer Diskussion das Problem beschrieben. Dieses ist das Grundproblem aller Managementsysteme zur Entscheidungsfindung.

Was ist aus der Sicht eines Managers dgentlich das Neue an TA? Wohl kaum die generelle Vorgehensweise, sondern ausschlie Blich die komplexere Zielfunktion, nach der optimiert werden muss. Zur Erläuterung möchte ich aus der VDI-Richtlinie Technikbewertung zitieren (VDI 1991):

„Technikbewertung bedeutet hier das planmäßige, systematische, organisierte Vorgehen, das

- den Stand einer Technik und ihre Entwicklungsmöglichkeiten analysiert,

- unmittelbare und mittelbare technische, wirtschaftliche, gesundheitliche, ökologische, humane, soziale und andere Folgen dieser Technik und möglicher Alternativen abschätzt,
- aufgrund definierter Ziele und Werte diese Folgen beurteilt oder auch weitere wünschenswerte Entwicklungen fordert,

- Handlungs- und Gestaltungsmöglichke iten daraus herleitet und ausarbeitet,

so daß begründete Entscheidungen ermö glicht und gegebenenfalls durch geeignete $\mathrm{In}$ stitutionen getroffen und verwirklicht we rden können."

Insbesondere der zweite Spiegelstrich in dieser Aufzählung legt die Verbindung von TA zum allseits akzeptierten Leitbild Nachhaltigkeit mit seinen bekannten Säulen nahe. Das führt zu der Aussage, TA ist das Konzept zur Operationalisierung des Leitbildes Nachhaltigkeit (Jischa 1997). Kurz: TA ist NachhaltigkeitsManagement.

An dieser Stelle möchte ich zunächst zwei in der Wirtschaft bekannte und etablierte Mhnagementsysteme skizzieren. Dabei beginne ich mit dem Qualitäts-Management nach der DIN/ISO 9000er Serie. Qualitätssichernde Maßnahmen hat es im produzierenden Gewerbe schon immer gegeben. Statistische Qualitätskontrollen waren lange Zeit Stand der Technik, bis durch Einführung der just-in-time Fertigung Lagerkapazitäten drastisch reduziert wurden. Durch das Verschwinden dieser Puffer mussten neue Maßnahmen zur Qualitätssicherung etabliert werden. Für statistische Qualitätskontrollen der (Vor-) Produkte fehlte nunmehr die Zeit, somit mussten Qualitätsprüfungen von Produkten verlagert werden auf die Prüfung, d. h. Zertifizierung, von Produzenten. Veränderte Abläufe in der Fertigung führten zu einem neuen Qualitäts-Managementsystem.

In der Produktentwicklung ist seit einigen Jahren das Qualitäts-Managementsystem nach DIN/ISO 9000 ff. prägend. Dabei werden die Anforderungen an ein Produkt in Regelkreisen mit zahlreichen Rückkopplungsschleifen verfolgt. Dies hat zu einer deutlichen Reduzierung der Entwicklungszeiten in der Industrie geführt. Zwingende Voraussetzung ist dabei, dass Produkteigenschaften formal quantifiziert werden.

Analog zum Qualitäts-Managementsystem ist (in der Regel von den Qualitäts-Experten) ein entsprechendes Umwelt-Managementsystem nach DIN/ISO 14000 ff. entwickelt worden. Dies beinhaltet im einzelnen Instrumente wie Environmental Auditing, Environ- 
mental Labeling, Environmental Performance Evaluation und Life-Cycle-Assessment (LCA), deutsch Ökobilanz genannt. Letztere ist innerhalb des Deutschen Instituts für Normung Gegenstand intensiver Standardisierungsbemühungen. In der folgenden Darstellung werde ich an beide Managementsysteme anknüpfen.

\section{Warum und wie findet TA in Unter- nehmen statt?}

Ausgehen möchte ich von der These, TA in der Wirtschaft als Nachhaltigkeits-Management zu verstehen. Dazu muss zunächst die Frage behandelt werden, warum Nachhaltigkeit ein Bestandteil des Zielsystems eines Unternehmens sein soll.

Seit einigen Jahren veröffentlichen Unternehmen ständig Umweltberichte, in denen $\mathrm{zl}^{-}$ mindest in den Vorworten die Verpflichtung zum Leitbild Nachhaltigkeit thematisiert wird. Eine ähnliche Selbstverpflichtung finden wir zunehmend auch in Geschäftsberichten sowie in öffentlichen Reden von Vorstandsvorsitzenden. Exemplarisch nenne ich hier das Einführungsreferat von J. E. Schrempp, Daimler Chrysler AG, anlässlich des ersten Weltingenieurtages, der vom VDI Verein Deutscher Ingenieure gemeinsam mit der EXPO $\mathrm{GmbH}$ als Auftaktveranstaltung zur EXPO im Juni 2000 in Hannover unter dem Motto „Globale Herausforderungen an die Ingenieure des 21. Jahrhunderts" durchgeführt wurde.

Erste Bekenntnisse zur Nachhaltigkeit sind aus der Chemischen Industrie zu verzeichnen gewesen. Beispielhaft möchte ich aus dnem Positionspapier des Verbandes der Chemischen Industrie zitieren, das zwei Jahre nach der Rio-Konferenz für Umwelt und Entwicklung veröffentlicht wurde (VCI 1994):

„Die zukünftige Entwicklung muss so gestaltet werden, dass ökonomische, ökologische und gesellschaftliche Zielsetzungen gleichrangig angestrebt werden.... Sustainability im ökonomischen Sinne bedeutet eine effiziente Allokation der knappen Güter und Ressourcen. Sustainability im ökologischen Sinne bedeutet, die Grenze der Belastbarkeit der Ökosphäre nicht zu überschreiten und die natürlichen Lebensgrundlagen $\mathrm{zu}$ erhalten. Sustainability im gesellschaftlichen Sinne bedeutet ein Höchstmaß an Chancengleichheit, Freiheit, sozialer Gerechtigkeit und Sicherheit."
Meines Wissens war H. Fürstenwerth an der Formulierung dieses Papieres maßgeblich beteiligt gewesen.

Man sollte Äußerungen dieser Art nicht mit dem Prädikat „Sonntagsreden“ abqualif izieren. Es sind handfeste wirtschaftliche Interessen, die die Repräsentanten der Wirtschaft zu derartigen Äußerungen veranlassen. Ich möchte dies am Beispiel der laufenden, teilweise auch stockenden (Folge-) Klimakonferenzen exemplarisch verdeutlichen, die aus unternehmerischer Sicht zu verschiedenen Konsequenzen führen bzw. führen können.

Zunächst sind veränderte Randbedingungen gesetzlicher und/oder fiskalischer Art zu erwarten. Beispielhaft seien $\mathrm{CO}_{2}$-Reduktionsziele und mögliche Instrumente wie Ökosteuer, wie Zertifikate und Lizenzen sowie joint implementation, kurz flexible Mechanismen, genannt. Weiter ist offenkundig, dass steigende Umweltschäden durch zunehmende Regenfälle, Überschwemmungen und Stürme die Rückversicherer alarmieren. Als Folge davon werden Versicherungsprämien an Risiko-Managementsysteme gekoppelt und sie erhalten dadurch eine ökonomische Qualität. Nicht zuletzt wird die Öffentlichkeit in Bezug auf Umweltfragen zunehmend sensibler. Kunden stellen kritische Fragen.

Alle diese Gründe lassen es mehr als grechtfertigt erscheinen, den „Umweltkosten“ zunehmende Aufmerksamkeit zu schenken. Im übrigen lebt die Wirtschaft ,nachhaltig“ von der Fähigkeit, zukünftige Probleme zu antizipieren.

Lange Zeit hat man die „klassischen“ betriebswirtschaftlichen Ziele und den Umweltschutz in Konkurrenz gesehen. Analog zu den Erfahrungen im Qualitäts-Management (Gute Qualität ist „,billiger“ als schlechte Qualität) hat sich im Umwelt-Management die Erkenntnis durchgesetzt, dass ,Ökologie = Langzeitökonomie" ist. Nur haben Betriebe eben ganz wesentlich auch eine Kurzzeit- neben der Langzeit-Perspektive. Umweltkosten entstehen, um zukünftige Schäden zu verhindern oder zu begrenzen, um Ressourcen zu erhalten und um zukünftigen wirtschaftlichen Nutzen zu stiften. Methodisch können Umweltkosten eingeteilt werden in solche für die Vermeidung, Verwertung und Beseitigung von Reststoffen (die betrieblichen Umweltkosten im engeren Sinne), 
durch nicht werterhöhenden Ressourceneinsatz sowie durch Produktverantwortung. Insbesondere der letzte Punkt wird zunehmend bedeutsam werden. Beispiele hierfür sind die Rücknahme von Altautos sowie Zwangspfand für Dosen u. ä..

Aus unternehmerischer Sicht ist es unverzichtbar, die Umweltkosten in bestehende Managementsysteme wie das Qualitäts- und das Umwelt-Managementsystem einzubauen. Denn nur ein Andocken an bestehende Systeme ist realistisch. So wie aus dem Qualitäts- das Umwelt-Management hervorgegangen ist, so wird das Risiko-Management folgen und alle werden in ein umfassendes NachhaltigkeitsManagementsystem einmünden.

Eine weitere für die Wirtschaft bedeutsame Anwendung in thematischer Nähe zur Vorgehensweise in der TA liegt in der Technologiefrüherkennung. Das wird auch der Grund dafür sein, dass das BMBF nunmehr den Begriff „Innovations- und Technikanalyse“ (ITA) bevorzugt, um TA mit der vorwiegend betriebswirtschaftlich betriebenen Innovationsforschung zu verschmelzen.

Technologiefrüherkennung wird in Forschung und Entwicklung dazu genutzt, schwache Signale neuer Technologien zu identifizieren, zu filtern und in FuE-Projekte umzusetzen. Technologiefrüherkennung ist ein zentrales Instrument des internen Innovations- und Technologie-Managements, mit dem Ziel, Reaktionszeiten und Entscheidungsspielräume für FuE-Entscheidungsträger zu beeinflussen.

Im Rahmen der Technologiefrüherkennung wird ein breites Methodenspektrum verwendet, etwa Literaturrecherchen, Patentanalysen, Kongressbeobachtungen, Expertengespräche oder Internetrecherchen. Aktuelle Methodenentwicklungen hin zu IT-basierten Verfahren, wie z. B. Data Mining, werden zu Effizienzsteigerungen führen.

Szenarioanalysen, Technologie-Roadmaps und Technologiebäume gehören zu den Filterungsmethoden von Technologieideen, die bei der Technologiefrüherkennung eingesetzt werden und einer Projektentscheidung in FuE vorausgehen.
Mit Hilfe der Technologiefrüherkennung besteht die Chance, Technologieoptionen möglichst frühzeitig zu erkennen und zu bewerten. Zur Realisierung einer solchen strategischen Ausrichtung muss der wirtschaftliche Nutzen einer frühen Überprüfung von Technologieideen hinsichtlich weitreichender Kriterien den Entscheidungsträgern vermittelt werden. Hierbei können Argumente wie das Erkennen von Marktpotenzialen und die rechtzeitige Absicherung durch Entwicklung und Patente, auch die Vermeidung von Risiken im ökologischen und sozialen Kontext dienen.

Aktuelle Managementmethoden, die weiche Faktoren wie Wissen oder Innovationen integrieren können, werden derartige Entwicklungen fördern. Beispielhaft sei das Instrument Balanced Scorecard (= ausgewogene Spielkarte) genannt. Damit wird ausgedrückt, dass der Erfolg eines Unternehmens nicht nur von rein monetären Kennzahlen abhängt. Erst zusammen mit weichen Faktoren wird daraus ein ,ausgewogener Berichtsbogen“. Dieses ist das betriebswirtschaftliche Pendant zu der durch die Globalisierung angestoßenen Diskussion „shareholder contra stakeholder-value“.

\section{$4 \quad$ Abschließende Bemerkungen}

Aus dem vorausgegangenen Abschnitt lassen sich mühelos etliche praxisrelevante und TAnahe Forschungsthemen herleiten. Welche Forschungsthemen aus der Sicht des Verfassers (eines Ingenieurs) formuliert werden können, ist an anderer Stelle beschrieben worden (Jischa 1999). Eine Auflistung daraus bislang entstandener Dissertationen und einer Habilitation ist soeben erfolgt (Jischa 2001). Die ehemaligen teilweise externen Mitarbeiter Decker, Frau Kensy, Ludwig, Nigge und Volkmar sind nach der Promotion bzw. Habilitation in die Wirtschaft gegangen oder haben, wie Poppe, aus der Wirtschaft heraus (Volkswagen AG) promoviert. In allen Fällen war die Beschäftigung mit wirtschaftsnaher TA ausschlaggebend, alle arbeiten weiterhin „TA-nah“. Das ist ein eindeutiger Beleg für das Interesse der Wirtschaft an TA, sofern diese wie geschildert praxisnah betrieben wird. 


\section{Literatur}

Jischa, M. F., 1997: Das Leitbild Nachhaltigkeit und das Konzept Technikbewertung. In: Chemie Ingenieur Technik 69, 12, S. $1695 \mathrm{ff}$

Jischa, M. F., 1999: Technikfolgenabschätzung in Lehre und Forschung. In: T. Petermann, R. Coenen (Hrsg.): Technikfolgen-Abschätzung in Deutschland. Frankfurt/New York: Campus Verlag, S. 165ff Jischa, M. F., 2001: Technikbewertung in der Lehre. In TA-Datenbank-Nachrichten Nr. 1, 10. Jahrgang, März, S. $61 \mathrm{ff}$

VCI / Verband der Chemischen Industrie e. V., 1994: Position der Chemischen Industrie.

VCI / Verband der Chemischen Industrie e. V., 1991: Technikbewertung - Begriffe und Grundlagen. VDI-Richtlinie 3780, Berlin

\section{Kontakt}

Prof. Dr.-Ing. Michael F. Jischa

Institut für Technische Mechanik der TU Clausthal Graupenstraße 3, D-38678 Clausthal-Zellerfeld

Tel.: + 49 (0) $5323 / 72-2083$

Fax: + 49 (0) $5323 / 72-2203$

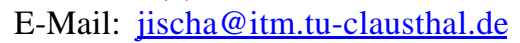

Internet:http://www.itm.tu-clausthal.de/

》

\section{Think different - just do it!}

\author{
von Hauke Fürstenwerth ${ }^{1}$, Leverkusen
}

Eine ernsthafte Erörterung des komplexen Themas „TA und Wirtschaft“ muss geführt werden auf der Basis klarer Vorstellungen, was TA ist, welche Bedeutung dem "Gemeinwohl" zugemessen wird und wer die handelnden Akteure in Wirtschaft und Technikentwicklung sind. Im nachfolgenden Beitrag wird am Beispiel des Silicon Valley verdeutlicht, welche herausragende Rolle Eigeninitiative und Kapitalmarkt bei der Entwicklung von Technik haben. Für eine TA, die als „Technik und Technikentwicklung reflektierende Forschung und Kommunikation" verstanden wird, ergeben sich aus der Praxis der Technikentwicklung in der Wirtschaft vielfältige Anknüpfungspunkte für Kooperationen.

\section{1}

\section{Prädiskursive Einverständnisse}

„TA, die sich selbst im Zentrum der gesellschaftlichen Zukunftsdiskussion verortet, kann aufgrund der Vielfalt und Heterogenität, ja oft geradezu Widersprüchlichkeit der einzelnen Felder keinesfalls auf standardisierte Verfahren und konsensuell definierte Problemlagen zurückgreifen.“ (Grunwald 2000, S. 133)

Auf der Basis eines derartigen Selbstverständnisses und eines solch weitreichenden Anspruches von TA, der, wie Weber und Schäffer zu Recht ausführen (Weber, Schäffer 2000, S. 155), an der Realität vorbei geht, halte ich es für ausgeschlossen, praktikable Ansätze für eine sinnvolle Interaktion von TA und Wirtschaft zu erschließen. Hierzu bedarf es konkreterer und realitätsbezogener Definitionen von TA. Basierend auf einer solchen Definition (Fürstenwerth 2000, S. 161) habe ich einen Vorschlag für eine strukturierte Kommunikation von Wirtschaft und Politik (Fürstenwerth 2000, S. 162) unterbreitet. Meiner Definition von TA und dem konkreten Vorschlag zu möglichen Interaktionen von TA und Wirtschaft habe ich einen Exkurs zu Technik, Wirtschaft und Gesellschaft vorangestellt, in welchem die große Bedeutung von Finanzwesen und Organisation für die Entwicklung von Technik herausgearbeitet wird. Aus welchem Grunde auch immer, es wird das Finanzwesen in TA Studien und Diskussionen stets ausgeblendet.

Den Beitrag von Axel Zweck nehme ich zum Anlass, der aktuellen Diskussion um TA und Wirtschaft einige weitere Klärungen voranzustellen, ohne die wiederum das Abgleiten in wohlgefällige Beliebigkeit droht. Es hat sich in den Sozialwissenschaften durchgesetzt, moderne Gesellschaften als ein Gebilde aus sich überlappenden (autonomen?) Teilsystemen zu beschreiben. Ich halte es jedoch für nicht zulässig, den Staat selbst als lediglich ein gesellschaftliches Teilsystem einzuordnen (Zweck 2001, S. 141). Eine klare Differenzierung Zw ischen Staat und Gesellschaft ist gerade im Kontext von TA und Wirtschaft unabdingbar. Der Staat mit seinen verfassungsgemäß definierten Institutionen, Verfahrensregeln und Gewalten bildet die für alle Teilsysteme der Gesellschaft verbindliche Grundlage. Das Cewaltmonopol sichert dem Staat die Möglich- 